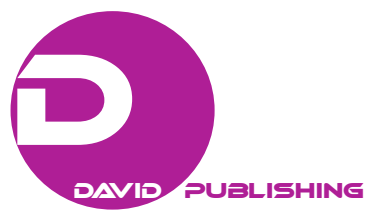

\title{
Lexical Dimensions Within the Register of Scientific Prose Style
}

\author{
Minoo Khamesian \\ NIT University, Babol, Iran
}

\begin{abstract}
Words, as we know, cannot be torn apart either from the concepts they express or from the functions they perform in each communicative context. In this regard, the unity of words and concepts is considered an indispensible part of all communication. Being a great hindrance on the way to efficient scientific communication, competence in general academic vocabulary seems to be of paramount importance. The reflection of the process of scientific work can be found in the general scientific vocabulary in order to establish a convenient, rational and simple system in this register. The present work focused on the lexical dimensions of scientific discourse in the hope to illuminate the path to bring about a better understanding of one of the crucial aspects of this register which seems to be overlooked while teaching EAP to non-native learners, a pragmatically oriented system which might provide a scientifically controlled "tool", to non-native learners.
\end{abstract}

Keywords: scientific register, general academic words, communicative competence

\section{Introduction}

The very nature of scientific prose style implies including a certain amount of the so-called "persuasive writing", using which the author would be capable of presenting his approach and/or suggest a commentary. Therefore, we can assume that the style cannot be confined to just the terms, although indispensable when introducing concepts and notions of a particular science. We cannot but also accept that terms would play a key role by virtue of their syntagmatic relationship with other terms and non-terms in the flow of speech.

Nonetheless, the term "word" would denote the basic unit of any language resulting from the association of a particular meaning with a particular group of sounds capable of a particular grammatical employment. Thus, it seems logical to say that a "word" is simultaneously a semantic, grammatical and phonological unit.

Effective and efficient scientific works tend to be clear and simple, as well as, accurate and, as mentioned, persuasive. In other words, since the purpose of science as a branch of human activity is to disclose by research the inner substance of things and phenomena of objective reality and to discover the laws regulating them, it should enable a researcher to predict, control and direct future developments to enhance both the material and social lives of humanity. Therefore, the main role of scientific prose is considered to be conveying factological information on different phenomena of the world surrounding us. In this regard, the language selected is responsible for this principle requirement (Goumovskaya, 1987).

Having said that, scientific communication cannot but be laden with difficulties of all kinds whenever the exchange of information is affected by different national languages. However, it is undeniable that even when the speakers use the same medium (e.g., English or Farsi), they are to agree on a certain variety or resister to

Minoo Khamesian, Dr./Ph.D in Linguistics, Basic Sciences, NIT University. 
serve as the main "tool of their trade". Thus, the concept of ESP in general, and EAP (the term used in this work) in particular can be related to a specific language code, i.e., the shared knowledge of linguistic conventions which would determine a given type of discourse, including the choice and arrangement of words, actually realized grammatical oppositions, the nature of syntactic constructions used and other linguistic features, as well.

Being sophisticated as a living register, EAP deals with how words can be used effectively in the course of communication. What demonstrated scientifically is considered a syllogism that can prove its conclusion by showing how it is necessarily capable of following from its explanatory principles (Kenny, 2004).

EAP with its emphasis on communicative competence as we know involves a consideration of how to establish contact, how to optimize the language to make it a more profitable means of exchanging information, how language as a system is used by scientists to communicate, etc. Thus, it is not a fallacy to say that EAP learners should be proficient in both the internal pattern of language as a self-contained system and how great a role these patterns play in the communicative operation of language in use. To further elaborate, the very concept of communicative competence presupposes the language user's potentials in expressing precisely what he intends to communicate applying devices appropriate to the given register, and undoubtedly his knowledge of rhetoric. As written time and again, scientific prose style as a mode of communicating findings is characterized by some features of rhetoric (such as "hedging devices"2), which are clearly manifested in scientific texts.

\section{Lexical Dimensions in Practice}

The vocabulary of our kind of English, as Akhmanova and Idzelis (1978) put, "an unvarnished medium" (p. 38), i.e., the prose which tends to be devoid of expressive evaluative overtones could be generally divided into three strata: general words, i.e., the most widely used, and most frequently occurring, in other words, the core of the language; special terms and terminological word combinations; and the so-called general scientific vocabulary, i.e., words most naturally used to ease communicating intellective information, regardless of being merely scientific pertaining to exact or natural sciences or connected with findings, observations and generalizations in the broader field of the Humanities.

As far as the general scientific words are concerned, they tend to be regarded as the main bearers of information transformation. However, none of the layers is a self-contained system inasmuch as there is permanent interaction and interchange between them (Khamesian, 2013).

What is of great importance is the semiotic principle (the law of the sign), which implies that the message is explicitly and unambiguously circulated in the related discourse community, scientific register here. Terms are most representative in this regard for they serve as units of a special language, e.g., technical, medical, etc. They can be coined for the exact expression of special notions and naming special objects ${ }^{3}$.

Turning to the other strata (general and general scientific), they can be compatible with the semiotic principle to a greater or less extent, which could mean that a stretch of speech may be clear and straightforward, or conversely, opaque. The latter would obviously contradict the established communicative orientation of EAP

\footnotetext{
1 Adapted from Akhmanova, O. S., \& Idzelis, R. F. What is the English We Use? Moscow: Moscow University Press, 1978.

2 Minoo Khamesian. Functional Peculiarities of Lexical Syntagm in Technical Writing//Advances in Social Sciences Research Journal, vol.4, N. 14, 2017.

${ }^{3}$ Minoo Khamesian, Rhetorical Impact through Hedging Devices in the 'Results and Discussion' Part of a Civil Engineering Research Article//Advances in Language and Literary Studies, 2015, vol. 6, N. 6, pp. 183-186.
} 
which is based on the function of message and calls for what Govishiani puts, "clarity of purpose" and "lucidity of exposition" (1992, p. 29).

To further illustrate the lexical stratification of a piece of "informative" writing, we now embark on analyzing a scientific-technical text which on the content plane presents a description of a phenomenon or concept from the domain of "pure" sciences. The text adduced below is on some issues of mechanical engineering ${ }^{4}$ :

Laser forming (LF) is one of the thermal forming processes, in which the laser beam irradiation causes localized thermal stresses. This leads to a permanent forming of metallic and non-metallic sheets without using mechanical forces and hard tools. Based on the scan path pattern, LF process is categorized into two groups of 2D and 3D forming. When a straight linear scan path is applied, workpiece experiences a simple bending as a $2 \mathrm{D}$ laser forming process. In $3 \mathrm{D}$ laser forming, workpieces are formed into a desired 3D shape when the scan path comprises a curved line or combined straight lines.

To begin with our analysis, concepts are considered the most general notions and categories of a given science, which, as truly scientific entities, come into being when certain words are firmly attached to them based on exact scientific definitions.

As clear, the general vocabulary is first and foremost represented by formal words, i.e., articles, conjunctions, prepositions, pronouns, numerals, needless to say, part and parcel of any text: the, $a$, which, of, one, into, two, etc. On the other hand, the terminological stratum comprises polylexemic terms, such as laser forming, thermal forming processes, laser beam irradiation, localized thermal stresses, metallic and non-metallic sheets. We can say what they have in common is syntactic construction of sorts based on which they could be called "loose compounds".

Furthermore, a number of units belong to the general scientific layer, e.g., apply, categorize, form, comprise, combine, etc. In actual fact, these units represent how separate words participate in the process of message communication. Otherwise stated, they would not remain on the "emic" level, but realize their colligational (morpho-syntactic) and collocational (lexical phraseological) potentialities in various combinations with units of the other layers (Akhmanova \& Gvishiani, 1979).

As noticeable, when terms (either mono or polylexemic) are combined with "general scientific words", they might be regarded as specific ultimate syntagmatic sequences, those particularly oriented towards a given branch of knowledge. Take, for example, the general scientific word "process" together with the polylexemic term "thermal forming", which create thermal forming processes.

With regard to the semiotic functioning of terms, they are considered based on the category of singularity, viz. the ability "to signify or signal exclusively what every one of them exist to mean or to dignify" (Akhmanova \& Idzelis, 1979, p. 27).

We could claim that scientific discourse tends to be a highly codified variety of linguistic usage with a great deal of uniformity in the realization of this specific mode of communication. According to Gasparian 1998, this specific language plays the role of a "shared code" between the communicators, which not only includes terms proper but various syntagmatic units as well. Thus, "ultimate syntagmatic sequences" are generally "encoded" and "decoded" globally without being articulated or further analysed into a succession of

\footnotetext{
4 Adapted from Tavakoli, A., Moslemi Naeini, H., Roohi, A. H., Hoseinpour Gollo, M., \& Imani Shahabad, S. Determining optimized radial scan path in $3 D$ laser forming of steelAISI 304 plates to produce bowl shapes. The International Journal of Advanced Manufacturing Technology, vol. 88, Ns. 5-8, 2017.
} 
smaller units, i.e., they might be overridden by the globality of the syntagmatic whole. It goes without saying that communication could be greatly facilitated when the members of a particular discourse community operate with readily comprehensible and easily decodable syntagmatic sequences.

Reconsidering general scientific vocabulary, as we can see there is a one-to-one correspondence between the plane of content and the plane of expression. They are the carriers of their literal meaning, devoid of additional expressive-emotional-evaluative overtones. It has always been assumed by and large that in intellective communication the author whose main objective is to pass on information would not rely on the power of words or their connotative values, hence the unity of words and concepts.

To communicate scientific findings, one should resort to easily decodable entities, which would require the unity of "reference" and "symbolism". To reiterate, we can observe a direct correspondence between content and expression so as to avoid ambiguity and misunderstanding.

\section{Conclusion}

When we speak of the unity of words and concepts, what we are primarily interested in is how to use our metalanguage, the tool of our trade, more effectively. Moreover, we should bear in mind that when what we are concerned with is communicative language teaching, we should move beyond the assumption that whenever language is used, people practise these or those syntactic patterns but aim at fulfilling certain communicative functions. Thus, before setting out to teach EAP, a clear-cut idea about this variety of English seems to be of paramount importance. EAP with its emphasis on communicative language learning requires a consideration of a host of issues, e.g., how to establish contact, how to optimize the language to make it a still more profitable means of exchanging information, etc. This means that learners should be proficient in both the internal patterns of language as a self-contained system and how the system relates to the communicative aspect of its use. If concept and notions are not brought together to form a system, there would be little hope that consistent and properly scientific definitions could be reached. We can add here that so much misunderstanding, malapropism, and other kinds of flagrantly unacceptable language can be due to the "authors" not having fully realized the extent of their imitative ability and the distance between themselves and the register they set out to follow.

\section{References}

Akhmanova, O. S., \& Gvishiani, N. (1979). ESP: Analysis through Synthesis. Fachsprache, vol. 1, Heft 4.

Akhmanova, O. S., \& Idzelis, R. F. (1978). What is the English we use? Moscow: Moscow University Press.

Gasparyan S. (1998). ESP as an object of learner-oriented teaching. Didaktic und Methodic des Fremdsprachenunterrichts. Heft I, Bonn.

Goumovskaya, G. (1987). Scientific prose style. In M. Khamesian (Ed.), Functional Approach to English for Engineering (with special reference to Iranian academic context). Lambert Publication; Online Book.

Gvishiani, N. (1992). Terminology as knowledge banks: The cognitive approach (with special reference to multilingual lexicography). EURALEX PROCEEDING I-II.TEMPERE.

Hazlitt, W. (1979). On familiar style. In O. Akhmanova, \& R. F. Idzelis (Eds.), Linguistics and semiotics. Moscow: Moscow University Press.

Kenny, A. (2004). A new history of western philosophy: Ancient philosophy. Oxford: Clarendon Press.

Khamesian, M. (2013). Functional approach to English for engineering (with special reference to Iranian academic context). Lambert Publication: Online Book.

\footnotetext{
5 Adapted from Akhmanova, O. Semantics. Processing of the XIIIth International Congress of Linguistics. Tokyo, 1982.
} 